Accepted for publication in the Astrophysical Journal

Preprint typeset using LTEX style emulateapj v. 11/10/09

\title{
MULTIPLE ABSORPTION-LINE SPECTROSCOPY OF THE INTERGALACTIC MEDIUM. I. MODEL
}

\author{
Yangsen YaO $^{1}$, J. Michael Shull ${ }^{1}$, Charles W. Danforth ${ }^{1}$, Brian A. Keeney ${ }^{1}$, And John T. Stocke ${ }^{1}$ \\ Accepted for publication in the Astrophysical Journal
}

\begin{abstract}
We present a physically-based absorption-line model for the spectroscopic study of the intergalactic medium (IGM). This model adopts results from Cloudy simulations and theoretical calculations by Gnat \& Sternberg (2007) to examine the resulting observational signatures of the absorbing gas with the following ionization scenarios: collisional ionization equilibrium (CIE), photoionization equilibrium, hybrid (photo- plus collisional ionization), and non-equilibrium cooling. As a demonstration, we apply this model to new observations made with the Cosmic Origins Spectrograph aboard the Hubble Space Telescope of the IGM absorbers at $z \sim 0.1877$ along the 1ES 1553+113 sight line. We identify Ly $\alpha, \mathrm{C}$ III, O vI, and N v absorption lines with two distinct velocity components (blue at $z_{b}=0.18757$; red at $\left.z_{r}=0.18772\right)$ separated by $\Delta(c z) /(1+z) \approx 38 \mathrm{~km} \mathrm{~s}^{-1}$. Joint analyses of these lines indicate that none of the examined ionization scenarios can be applied with confidence to the blue velocity component, although photoionization seems to play a dominant role. For the red component, CIE can be ruled out, but pure photoionization and hybrid scenarios (with $T<1.3 \times 10^{5} \mathrm{~K}$ ) are more acceptable. The constrained ranges of hydrogen density and metallicity of the absorbing gas are $n_{\mathrm{H}}=(1.9-2.3) \times 10^{-5} \mathrm{~cm}^{-3}$ and $Z=(0.43-0.67) Z_{\odot}$. These constraints indicate $\mathrm{O}$ VI and $\mathrm{H}_{\mathrm{I}}$ ionization fractions, $f_{\mathrm{OVI}}=0.10-0.15$ and $f_{\mathrm{HI}}=(3.2-5.1) \times 10^{-5}$, with total hydrogen column density $N_{H}=(0.7-1.2) \times 10^{18} \mathrm{~cm}^{-2}$. This demonstration shows that joint analysis of multiple absorption lines can constrain the ionization state of an absorber, and results used to estimate the baryonic matter contained in the absorber.
\end{abstract}

Subject headings: Cosmology: observations — intergalactic medium — quasar: absorption lines — ultraviolet: general

\section{INTRODUCTION}

The intergalactic medium (IGM) is believed to be the main reservoir of baryons throughout the history of the universe (e.g., Shull 2009). In the early universe $(z>3)$ the IGM was dominated by photoionized gas traced by Ly $\alpha$ forest lines (e.g., Rauch et al. 1997). At $z \lesssim 2$, the IGM is expected to be a mixture of photoionized and collisionally ionized gas produced by radiation of active galactic nuclei (AGN) and gravitational shock heating during the formation of galaxies, groups, and clusters (e.g., Birnboim \& Dekel 2003; Kereš et al. 2009). Cosmological hydrodynamic simulations of large-scale structure formation predict that, at the current epoch, $\gtrsim 40 \%$ of the baryons may exist in the form of a warm-hot intergalactic medium (WHIM) at temperatures $T \sim 10^{5}-10^{7} \mathrm{~K}$ in a "Cosmic Web" distributed between the galaxies (e.g., Cen \& Ostriker 2006; Oppenheimer \& Davé 2008).

Conducting a complete baryon inventory for the current universe is one of the major tasks of modern cosmology. The predicted physical conditions in the hottest parts of the WHIM may require detection using X-ray absorption lines (e.g., Davé et al. 2001; Cen \& Fang 2006). However, owing to limited sensitivity and spectral resolution, X-ray observations have so far yielded only a few controversial detections (e.g., Fang et al. 2002, 2007; Nicastro et al. 2005; Kaastra et al. 2006; Buote et al. 2009). Current X-ray observatories such as Chandra and XMM-Newton have spectral resolution $(\lambda / \Delta \lambda \sim 400)$ and detection limits $\left(\log N_{\mathrm{OVII}} \gtrsim 15.5\right.$; Yao et al. 2009, 2010). Instruments operating in the far- and

\footnotetext{
${ }^{1}$ Center for Astrophysics and Space Astronomy, Department of Astrophysical and Planetary Sciences, University of Colorado, 389 UCB, Boulder, CO 80309; yaoys@ colorado.edu
}

near-ultraviolet (UV) wavelength bands have superior spectral resolution, $\lambda / \Delta \lambda \sim 20,000-43,000$. With the larger intrinsic line strengths (O vI vs. O VII) and the higher spectral resolution and sensitivity, the UV spectrographs aboard the Hubble Space Telescope (HST) - the Space Telescope Imaging Spectrograph (STIS) and the Cosmic Origins Spectrograph (COS) - are sensitive to lower column densities, $\log N_{\text {OVI }} \gtrsim 12.7$. These instruments can probe the WHIM up to redshifts $z \lesssim 0.7$ (e.g., Danforth \& Shull 2005, 2008; Savage et al. 2005; Tripp et al. 2008; Thom \& Chen 2008). The major advances in low-redshift IGM studies in the next decade are expected to come from archival data from STIS and the Far Ultraviolet Spectroscopic Explorer (FUSE) together with new observations from COS. Indeed, the multiphase nature of the IGM has been revealed through detected absorption lines of ions at various ionization states (e.g., H I, C III, C Iv, Si III, Si Iv, N v, O vi, and Ne vIII) by FUSE, STIS, and COS. In order to proceed, we need to know how to convert measurement of these absorption lines to amounts of baryonic matter, which depends on ionization fractions, metallicity, and ionization phases of the IGM (Penton et al. 2004; Tripp et al. 2008; Danforth \& Shull 2008, hereafter DS08). None of these properties is well measured for many absorbers, and in many cases the data do not permit such determinations.

Spectroscopy of multiple absorption lines provides a powerful diagnostic of IGM properties. These diagnostics have been applied to many systems by measuring ionic column densities and doppler velocities, examining the significance of photo-, collisional, and non-equilibrium ionization, and assessing the physical conditions in the absorbers (e.g., Richter et al. 2004; Savage et al. 2005; Tripp et al. 2008; Narayanan et al. 2010). However, because these applications follow a multistep procedure, the derived physical parame- 
ters could depend in complex ways on the initially measured quantities (e.g., temperature and metallicity of an intervening gas could co-vary as different functions of $\mathrm{O}$ VI column density), which makes estimations of their uncertainty hard to implement. We present an absorption-line model in which the physical properties of the absorber have been used as the primary fitting parameters. Spectral fitting of multiple absorption lines with this model will not only yield constraints to these parameters but automatically take care of covariance of all parameters. As a case study, we apply this model to an interesting and well-defined absorption system at $z \sim 0.1877$ along the sight line of 1ES $1553+113$ observed with COS.

The paper is organized as follows. We describe our COS observations and data reduction procedure in $\S 2$, and introduce our spectral model in $\S 3$. We apply our model to the observations and discuss our results and their implications in $\S 4$

\section{OBSERVATIONS AND DATA REDUCTION}

One of the Guaranteed Time Observation (GTO) targets, the BL Lacertae Object 1ES 1553+113 was observed on 2009 September 22 with COS, which was installed on the HST during Hubble Servicing Mission 4. The observations were taken with the same FP_POS position (FP_POS=3) but with different central wavelengths, shifting along the sequence $1291 \AA$, $1300 \AA, 1309 \AA$, and $1318 \AA$ for the G130M grating (covering $1134 \AA$ to $1465 \AA$ ), and $1589 \AA, 1600 \AA, 1611 \AA$, and $1623 \AA$ for the G160M grating (covering $1383 \AA$ to $1797 \AA$ ). The total exposure times are $3109.6 \mathrm{~s}$ with the G130M and $3802.8 \mathrm{~s}$ with the G160M.

The observations were calibrated with the pipeline CALCOS (version 2.11f). Flat-fielding, alignment, and coaddition of the processed exposures were carried out using IDL routines developed by the COS GTO team specifically for COS FUV data2 Briefly, true flat-fielding of COS observations has yet to be developed, but each exposure was corrected for narrow, $\sim 15 \%$-opaque shadows from repellor grid wires. The one-dimensional map of grid-wire opacity was shifted from detector coordinates to wavelength space and divided from the reduced one-dimensional flux. Within the same grating (G130M or G160M), these spectra were then cross-correlated and combined to form an exposure-weighted coadded spectrum (See Danforth et al. 2010b for a detailed description of observations and data reduction).

In the COS spectra of 1ES $1553+113$, three absorption systems were identified near redshift $z=0.187$ (Danforth et al. $2010 \mathrm{~b})$. Among these systems, the one at $z \sim 0.1877$, as presented in Figure 1, has the most significant detections of absorption lines of Ly $\alpha, \mathrm{C}$ III, and the $\mathrm{O}$ VI and $\mathrm{N} v$ doublets. We therefore use this system to test our absorption-line model.

\section{MODELS}

We considered intergalactic absorbers in the following ionization model conditions: (1) collisional ionization equilibrium (CIE; Case 1); (2) photoionization equilibrium (PE; Case 2); (3) medium with a constant electron temperature illuminated by the UV photoionizing background (hybrid model; Case 3); and (4) post-shock gas undergoing non-equilibrium

\footnotetext{
${ }^{2}$ See http: //casa.colorado.edu/ danforth/costools.htm for our coaddition and flat-fielding algorithm and additional discussion.
}

TABLE 1

IONIZATION SCENARIOS

\begin{tabular}{ll}
\hline \hline \multicolumn{1}{c}{ Scenarios } & Free Parameters \\
\hline Case 1 : collisional ionization equilibrium (CIE) & $T, Z, N_{\mathrm{H}}$ \\
Case 2 : pure photoionization equilibrium (PE) & $n_{\mathrm{H}}, Z, N_{\mathrm{H}}$ \\
Case 3 : photo- plus collisional ionization (hybrid) & $n_{\mathrm{H}}, T, Z, N_{\mathrm{H}}$ \\
Case 4 : non-equilibrium isobaric cooling & $T, Z, N_{\mathrm{H}}$ \\
Case 5 : non-equilibrium isochoric cooling & $T, Z, N_{\mathrm{H}}$ \\
\hline
\end{tabular}

Note. - Cases 1, 4, and 5 are based on results by Gnat \& Sternberg (2007), and Cases 2 and 3 are based on Cloudy simulations. $n_{\mathrm{H}}, T$, $Z$, and $N_{\mathrm{H}}$ are the number density, temperature, metallicity, and total column density of the absorbing gas, respectively.

isobaric (Case 4) or isochoric (Case 5) cooling. These five cases represent the most explored ionization scenarios in ultraviolet spectroscopic studies of the IGM.

For Cases 1, 4, and 5, we adopted the results of Gnat \& Sternberg (2007), in which they calculated the ionic abundances for gas in CIE state and for a shock-heated gas cooling from $\log T=6.7$ either at constant density (isochorically) or at constant pressure (isobarically).

For Case 3, we ran Cloudy (version 08.00; Ferland et al. 1998) simulations with parameters of temperature $(T)$ and hydrogen number density $\left(n_{\mathrm{H}}\right)$. A 100 -kpc thick slab (e.g., Davé et al. 2001; Penton et al. 2004) was used to approximate an intergalactic absorbing cloud, which is illuminated by the intergalactic ionization field of Haardt \& Madau (2001) at redshifts $z=0-13$ with a step size of 0.1 . In our spectral fitting described below, the estimated ionization field at the closest redshift bin to that of the observed absorber was utilized. The grids of $\log T$ and $\log \left[n_{\mathrm{H}}\left(\mathrm{cm}^{-3}\right)\right]$ were 4 to 7.5 and -6 to -2 , respectively, with a step size of 0.1 for each grid. The collisional ionization effects were included by setting a constant electron temperature in the simulations. The relative ionic column densities were initially calculated with the metallicity $\log \left(Z / Z_{\odot}\right)=-1$, and the results for metal (heavier than helium) ions were then scaled to different metallicities. We took the results with $\log T=4$ for a pure photoionization model (Case 2).

Table 1 summarizes these ionization scenarios, and Figures 2 and 3 demonstrate the differences in $\mathrm{O}$ VI and $\mathrm{N} \mathrm{V}$ ionization fractions among different models. In any of these models, for a given set of physical parameters (e.g., $Z, T$, and/or $n_{\mathrm{H}}$ ), there will be a determined set of column density ratios among all ions. In turn, if the observed multiple absorption lines occur in the same gas undergoing the same ionization scenario, the column density ratios among metal ions will provide constraints on $T$ and/or $n_{\mathrm{H}}$, and ratios between metal ions and $\mathrm{H}$ i will yield metallicity diagnostics of the absorbing gas.

We developed a model that can be used to jointly analyze multiple absorption lines to realize these diagnostics. The model, which is revised from our X-ray absorptionline model absline (Yao \& Wang 2005), is summarized as follows. An absorption-line profile can be described as (Rybicki \& Lightman 1979):

$$
f(\lambda)=f_{c}(\lambda) \exp [-\tau(\lambda)],
$$

where $f_{c}$ is continuum flux and $\tau$ is the absorption opti-

3 We in fact used the command "HM05" in Cloudy simulations to specify both shape and flux of the ionization field; the normalization of the field evolves from $1.6 \times 10^{-6} \mathrm{erg} \mathrm{s}^{-1} \mathrm{~cm}^{-2}$ at $z=0$ to $1.3 \times 10^{-5} \mathrm{erg} \mathrm{s}^{-1} \mathrm{~cm}^{-2}$ at $z=1$. 


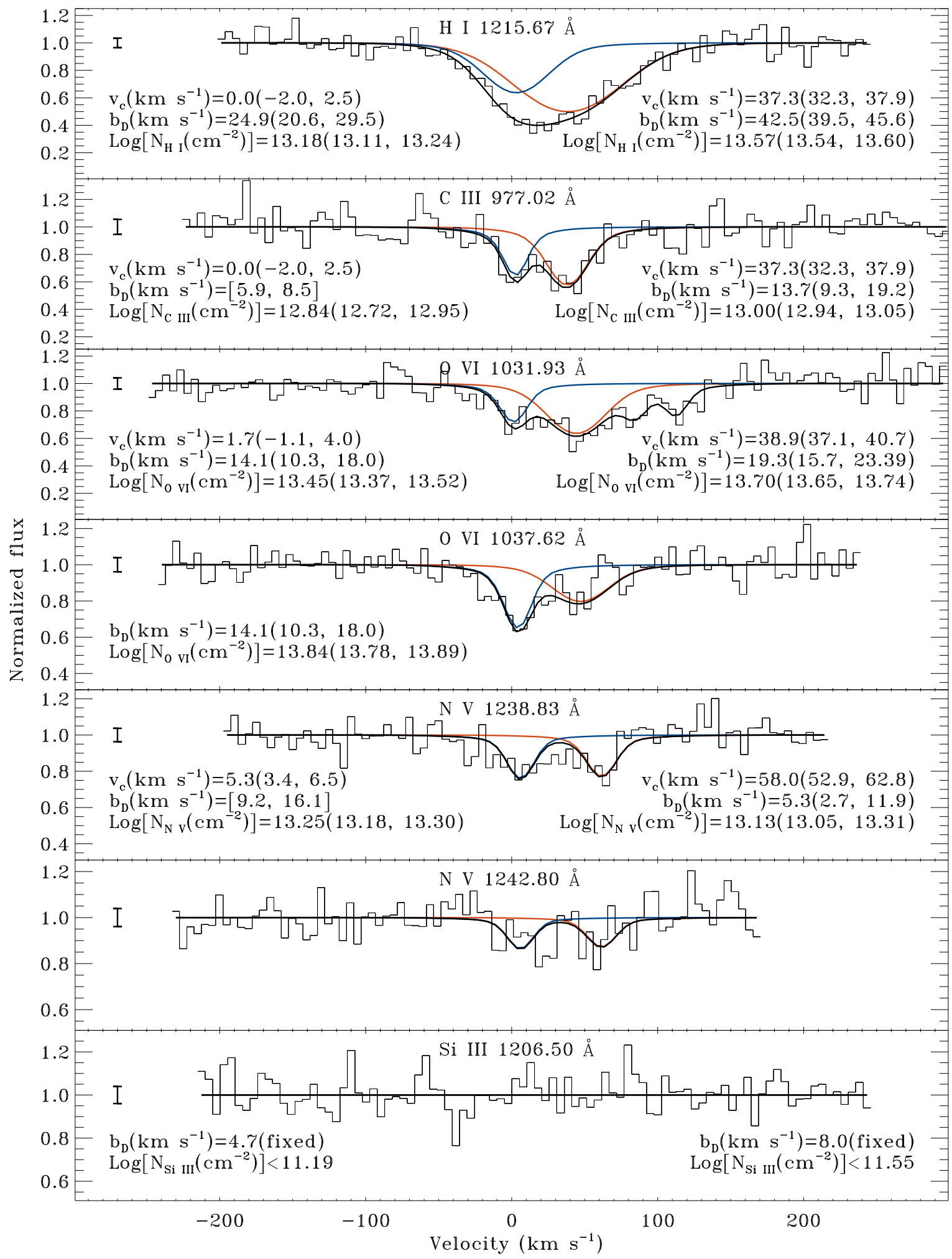

FIG. 1.- Absorption lines at $z \sim 0.1877$ detected in the COS spectrum of 1ES $1553+113$. The vertical error bar shown at $-270 \mathrm{~km} \mathrm{~s}^{-1}$ in each panel indicates the averaged flux error in the plotted wavelength range. Thick black lines show the continua and total absorption, and blue and red lines mark fits to the two velocity components, at $z_{b}=0.18757$ and $z_{r}=0.18772$. The labeled line shifts $\left(v_{\mathrm{c}}\right)$, doppler velocities $\left(b_{\mathrm{D}}\right)$, and column densities $\left(N_{\mathrm{i}}\right)$ are measured relative to $z=0.18757$. Numbers in brackets "[ $]$ " are calculated values with respect to other ions. Doublet lines are jointly measured when applicable. Data are binned by a factor of 2 with $\sim 3$ bins in each resolution element. See text for details. 


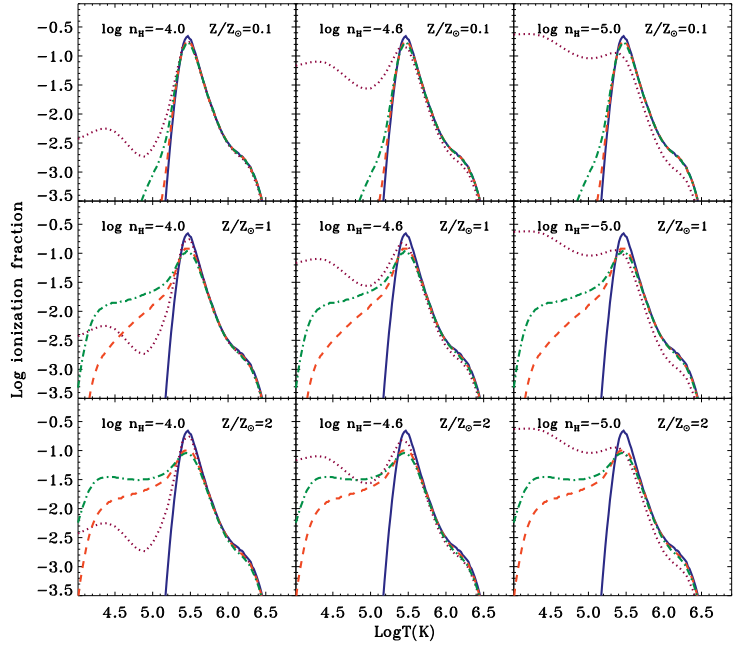

FIG. 2.- Ionization fraction of O VI under different physical conditions. The solid-blue, dashed-red, dash-dotted-green, and dark-red-dotted curves indicate the CIE, Isobaric, Isochoric, and Hybrid models, respectively. For the hybrid model, plots show the fraction in gas illuminated by the photoionization field at $z=0$.

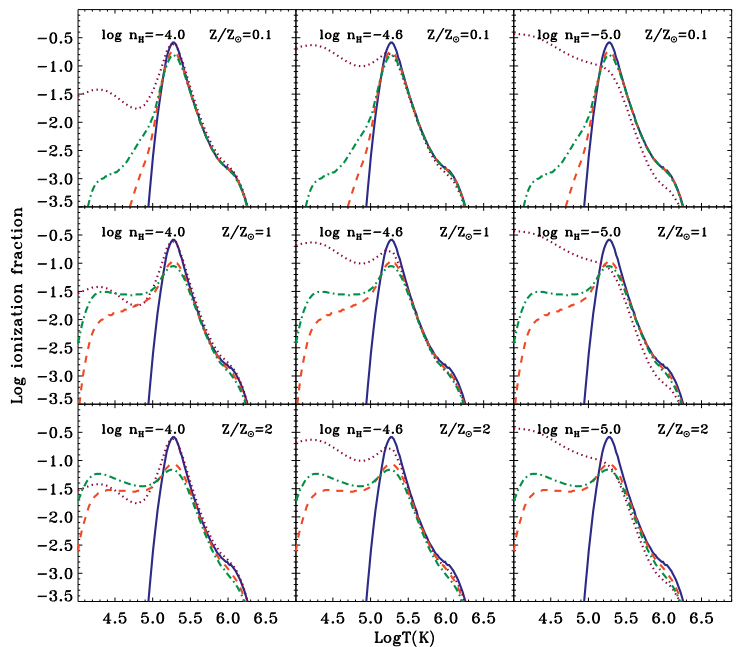

FIG. 3.- Same as Figure 2] but for ion N V.

cal depth, given by a Voigt function depending on wavelength $(\lambda)$, column density $\left(N_{i}\right)$, doppler width $\left(b_{D}\right)$, oscillator strength $\left(f_{l u} ; l\right.$ and $u$ are the lower and upper excitation levels for the transition), and the natural damping factor $(\gamma)$ of the transition. For a set of input parameters $\left(N_{\mathrm{HI}}, Z, T\right.$, and/or $\left.n_{\mathrm{H}}\right)$ of any ionization case discussed above, the model queries the simulation and/or calculation results and interpolates the values lying between the simulated and calculated grids to obtain a unique value for $N_{i}$. These physical properties are fitting parameters linked together in a joint analysis of multiple lines.

We implement our spectral analysis within the software package Xspec (Arnaud 1996), to utilize its $\chi^{2}$ minimization algorithm over multiple fitting variables, where

$$
\chi^{2}=\sum_{i}\left(\frac{f_{i}^{o}-f_{i}^{p}}{\sigma_{i}}\right)^{2} .
$$

Here, for each wavelength bin $(i), f_{i}^{o}, f_{i}^{p}$, and $\sigma_{i}$ are the observed flux, the model-predicted flux, and the uncertainty of

\footnotetext{
${ }^{4}$ http://heasarc.nasa.gov/docs/xanadu/xspec/
}

$f_{i}^{o}$. For COS gratings G130M and G160M, we interpolate the line spread functions (LSFs) to the desired wavelength grid and then use the IDL scripts wrt_ogip_rm and writeph 7 to transform these LSFs and the coadded spectra obtained in $\S 2$ to a "fits" format for input to Xspec. In spectral fitting, the spectral models are automatically convolved with these wavelength-dependent LSFs.

In the following, we apply this model to the absorption system at $z=0.187$ along the sight line to $1 \mathrm{ES} 1553+113$. Because velocity components in this system can clearly be resolved (Fig. 1), the spectral analyses conducted here are similar to traditionally employed profile-fitting techniques, although our physical variables are the fitting parameters. When several velocity components are severely blended, arbitrarily resolving components through spectral fitting could lead to unreliable results.

\section{SPECTRAL FIT, RESULTS, AND DISCUSSION}

Using our absorption-line model, we first fit the lines individually to obtain properties of the absorbing ions. The local continua are interpolated from the best fits to spectral ranges $\pm 150 \mathrm{~km} \mathrm{~s}^{-1}$ from line centroids. Absorption lines of C III, $\mathrm{O}$ vI, and $\mathrm{N} v$ clearly show two velocity components (blue and red) in the $z \sim 0.1877$ absorber, but component separation is not obvious in the broad $\operatorname{Ly} \alpha$, other than asymmetry in the red wing (Figure 1). In both components, centroids of the metal lines are well aligned, except for the red $\mathrm{N} v$ that deviates $\sim 20 \mathrm{~km} \mathrm{~s}^{-1}$ from others. We therefore set the centroid velocities of the two $\mathrm{H}$ i components equal to those of the corresponding $\mathrm{C}$ III absorption. Fitting these lines individually yields the velocity shift $v_{c}, b_{D}$, and $N_{i}$ of the absorbing ions. For each velocity component, we link $b_{D}, N_{i}$ and the centroids of the $\mathrm{O}$ VI and $\mathrm{N} v$ doublets to get better constraints (except the blue component of O vI). The O vi $1037.62 \AA$ line should be about half the strength of O vi $1031.93 \AA$, but it appears stronger in the blue component. This mismatch is likely caused by an unidentified weak intergalactic Ly $\alpha$ absorber at $z=0.01364$ that contaminates the blue component of O vi $1037.62 \AA$. We therefore only link the $b_{D}$ and velocity centroids and vary $N_{\mathrm{OVI}}$ independently in the doublet. We also exclude this line in our joint analysis of multiple absorption lines described below. The red wing of the $1031.93 \AA$ line is blended with $\operatorname{Ly} \beta$ absorption at $z=0.1950$. We use a two-velocity model to fit the $\mathrm{Ly} \beta$ profile and fix its contribution in our analysis (Fig. 1). The $b_{D}$ of $\mathrm{C}$ III and $\mathrm{N} v$ cannot be constrained in the blue component, owing to the narrowness of the $\mathrm{C}$ III line and the poor $\mathrm{S} / \mathrm{N}$ around the $\mathrm{N} v$ line. We set the $b_{D}$ ranges for $\mathrm{C}$ III and $\mathrm{N} v$ to those of $\mathrm{H}$ I and $\mathrm{O}$ VI assuming thermal broadening. Because there is no visible Si III $1206 \AA$ line in the spectrum (see Danforth et al. 2010b), we fix the line centroid velocities to those of $\mathrm{C}$ III and $b_{D}$ to the corresponding thermal values of $\mathrm{H}$ i to obtain upper limits on $N_{\text {SiIII }}$. The constrained values of $v_{c}, b_{D}$, and $N_{i}$ are labeled in Figure 1 .

Next, we jointly analyze these absorption lines in each velocity component to examine the five ionization scenarios discussed in $\S 3$ Line widths of IGM absorbers have been shown to possess significant contributions from turbulent motion or unresolved components; measured widths there-

\footnotetext{
5 http://www.stsci.edu/hst/cos/performance/spectral_resolution/

${ }^{6} \mathrm{http} / / /$ hea-www.harvard.edu/PINTofALE/pro/util/

${ }^{7}$ http://astro.uni-tuebingen.de/software/idl/aitlib/fits/
} 
fore provide upper limits on thermal broadening in the absorber (Thom \& Chen 2008; Tripp et al. 2008; Danforth et al. 2010a). Attributing the constrained $b_{D}$ to purely thermal broadening, we derive upper limits of $\log T \leq 4.7$ and 5.1 for the absorbing gas in Cases 1 and 3-5 (both are based on $\mathrm{H} \mathrm{I}_{\mathrm{I}}$ measurements) for blue and red components respectively. We apply the upper limits in our joint analyses, and constrain the physical properties $\left(Z, T, n_{\mathrm{H}}\right)$ and total hydrogen column density $N_{\mathrm{H}}$ of the absorbing gas (Table 2). Given the constrained values of $Z, T, n_{\mathrm{H}}$, the column density of any ion can be calculated. The predicted column densities $\log N_{\text {SiIII }}=8.82-11.16$ for all acceptable scenarios, consistent with the upper limits derived from the data (Figure1, Danforth et al. 2010b).

The spectral fitting indicates that some ionization scenarios can be ruled out by the data. To quantify how well these scenarios fit the velocity components, we record the difference $\left(\delta \chi^{2}\right)$ of their best-fit $\chi^{2}$ from those of fitting these lines individually. According to an F-test, values $\delta \chi^{2}<0.1,1.0$, and 4.0 indicate that a joint fit is identical to an individual-line fit at $>94 \%(99 \%), 55 \%(75 \%)$, and $9 \%(19 \%)$ confidence levels for Case 3 (Cases 1, 2, 4, 5), respectively. In this work, a joint fit is considered acceptable if it is identical to the individualline fit at $>10 \%$ confidence level. None of the examined ionization scenarios is acceptable for the blue component, although photoionization seems to play an important role in the absorbing gas. The non-fruitful exercise for the blue component suggests that the low and high ions are not produced in the same absorber in the same ionization conditions (see discussion below). As an alternative, non-equilibrium cooling plus photoionization could reconcile all the measurements (J. Li 2010, private communication). Unfortunately, this scenario was not calculated in Gnat \& Sternberg (2007). For the red component, PE and hybrid ionization cases (Cases 2 and 3 ) are acceptable at $>75 \%$ confidence levels (Table 2), the non-equilibrium cooling scenarios (Cases 4 and 5) are less acceptable, and the CIE scenario (Case 1) can be ruled out.

Besides the ion column density ratios, differences in thermal broadening of absorption lines of different elements also constrain the gas temperature. We assume that the line broadening consists of both thermal and non-thermal (turbulent) broadening, related by $b_{D}^{2}=\left(b_{\text {ther }}^{2}+b_{\text {turb }}^{2}\right)$. Taking $k T=1 / 2 m b_{\text {ther }}^{2}$, we can use the measured $b_{D}$ in different elements to determine $T, b_{\text {ther }}$, and $b_{\text {turb }}$. For the red component, measurement of $\mathrm{H} \mathrm{I}_{\mathrm{I}}$ and $\mathrm{C}$ III (Fig. 11) yields $4.9<\log T<5.1$ and $0 \mathrm{~km} \mathrm{~s}^{-1}<b_{\text {turb }}<16 \mathrm{~km} \mathrm{~s}^{-1}$, while the combination of $\mathrm{H}$ I and $\mathrm{O}$ vi yields $4.8<\log T<5.1$ and $11 \mathrm{~km} \mathrm{~s}^{-1}<b_{\text {turb }}<$ $22 \mathrm{~km} \mathrm{~s}^{-1}$. These constraints (particularly on temperatures) are consistent with those obtained from the column density ratios (Table2).

In our analysis, we assumed that both low and high ions were produced in a co-spatial absorber with the same ionization conditions. This assumption is often justified by the similarity in their absorption-line kinematics (Figure 1). However, it has also been suggested (DS08; Narayanan et al. 2010) that these ions may be multi-phase (non co-spatial), with the low ions (e.g., $\mathrm{H}$ I, C III, Si III) arising from cool-phase photoionized gas and the high ions (e.g., $\mathrm{O}$ vi and $\mathrm{N}$ v) arising primarily from collisional ionization. Such scenarios could occur, for instance, in IGM filaments with photoionized cores surrounded by infalling shock-heated gas (Danforth \& Shull 2008). To examine this possibility, we use the PE model (Case 2) to jointly fit the blue $\mathrm{H} \mathrm{I}, \mathrm{C}$ III, and Si III lines where all our models failed in joint analyses of high and low ion lines (Ta-

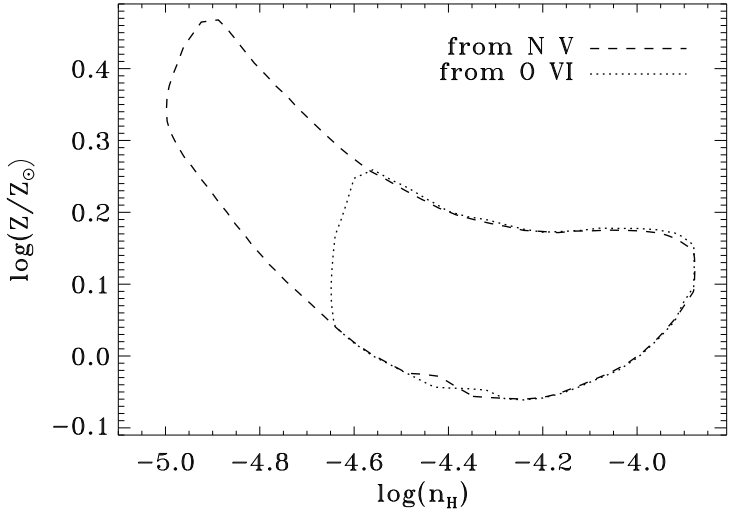

FIG. 4.- The $1 \sigma$ co-varying contours of $\log \left(Z / Z_{\odot}\right)$ vs. $\log n_{H}$ for photoionization model, obtained by jointly fitting the blue $\mathrm{H} \mathrm{I}, \mathrm{C}$ III, and Si III lines and using the observed amount of O VI (dotted) and N V (dashed) as upper limits.

ble 2). Because we have only two detections ( $\mathrm{H}$ I and $\mathrm{C}$ III) and one upper limit ( $\mathrm{Si}$ III) and there are three free parameters $\left(n_{H}\right.$, $Z$, and $N_{H}$; Table 2), we cannot obtain meaningful constraints on these parameters. Requiring the amount of $\mathrm{O}$ VI and $\mathrm{N} \mathrm{v}$ produced in the PE ionization to be less than those observed, $\log N_{\mathrm{OVI}}<13.52$ and $\log N_{\mathrm{NV}}<13.30$ (Fig.11), we obtain the $1 \sigma$ co-varying contour of $\log \left(Z / Z_{\odot}\right)$ vs. $\log n_{H}$ (Fig. (4). The overlapped region in Figure 4 indicates the constrained ranges of $\log n_{H}=(-4.65,-3.87)$ and $\log \left(Z / Z_{\odot}\right)=(-0.65,0.25)$ for the cooler PE ionization gas. While the amount of $\mathrm{O}$ vi and $\mathrm{N} v$ contained in the PE ionized gas is still poorly constrained, it is hard to use the remaining amount to further constrain the properties of the hotter collisional gas. Once multiple ionization states of low and high ions are available, we can use this method to constrain properties of different phases and probe the interactions between them.

All acceptable scenarios require the metallicities of the absorbing gas to be $\gtrsim 40 \%$ of the solar value (Table 2). Such high metallicities suggest that we are sampling enriched intragroup gas or a circumgalactic medium with a small impact distance to a galaxy (e.g., Jenkins et al. 2005; Aracil et al. 2006; Pratt et al. 2007). A deep galaxy survey along the 1ES 1553+113 sight line (B. Keeney et al. 2011, in preparation) will examine these possibilities and suggest a physical origin for these absorbers. As the metallicity increases, the cooling times will become shorter compared to the recombination time. The absorbing gas could then become an "overcooled" plasma in which the high ions ( $\mathrm{C}$ Iv, $\mathrm{N} \mathrm{v}, \mathrm{O}$ vi) are no longer good indicators of the gas temperature, especially in cooling models (Gnat \& Sternberg 2007). In this case, temperature diagnostics can still be obtained from the differential temperature dependence between low ions (e.g., $\mathrm{H}$ I and C III) and high ions, if they are co-spatial, or from differential thermal broadening of different elements.

We assumed relative solar metal abundances of Asplund et al. (2009) in our spectral analysis. This assumption can be relaxed when absorption lines from different ionization states of the same elements are available to constrain the physical parameters of the absorber. Spectroscopy of multiple absorption lines from different elements will then yield their relative abundances.

The joint analyses presented in this work also enable us to directly estimate the total baryon content of Ly $\alpha$ and metalline absorbers and the uncertainty in those measurements. The primary systematic uncertainties arise from modeling the metallicity and ionization fractions. Since our model uses 
TABLE 2

DiAgNOSTIC RESULTS

\begin{tabular}{|c|c|c|c|c|c|c|c|c|c|c|}
\hline & \multicolumn{5}{|c|}{ Blue component $z_{b}=0.18757$} & \multicolumn{5}{|c|}{ Red component $z_{r}=0.18772$} \\
\hline & $\delta \chi^{2}$ & $\begin{array}{l}\log n_{\mathrm{H}} \\
\left(\mathrm{cm}^{-3}\right)\end{array}$ & $\begin{array}{c}\log T \\
(\mathrm{~K})\end{array}$ & $\log \left(Z / Z_{\odot}\right)$ & $\begin{array}{l}\log N_{\mathrm{H}} \\
\left(\mathrm{cm}^{-2}\right)\end{array}$ & $\delta \chi^{2}$ & $\begin{array}{l}\log n_{\mathrm{H}} \\
\left(\mathrm{cm}^{-3}\right)\end{array}$ & $\begin{array}{c}\log T \\
(\mathrm{~K})\end{array}$ & $\log \left(Z / Z_{\odot}\right)$ & $\begin{array}{l}\log N_{\mathrm{H}} \\
\left(\mathrm{cm}^{-2}\right)\end{array}$ \\
\hline Case 1 & $>100$ & NA & {$[<4.7]$} & $x$ & $x$ & $>100$ & NA & {$[<5.1]$} & $x$ & $x$ \\
\hline Case 2 & 21.5 & $-4.69_{-0.04}^{+0.04}$ & NA & $0.05_{-0.08}^{+0.09}$ & $17.51_{-0.06}^{+0.05}$ & 0.8 & $-4.74_{-0.03}^{+0.03}$ & NA & $-0.22_{-0.05}^{+0.05}$ & $17.89_{-0.03}^{+0.03}$ \\
\hline Case 3 & 16.6 & $-4.62_{-0.08}^{+0.05}$ & {$[<4.7]$} & $0.07_{-0.09}^{+0.07}$ & $17.57_{-0.08}^{+0.09}$ & 0.1 & $-4.67_{-0.09}^{+0.03}$ & {$[<5.1]$} & $-0.24_{-0.12}^{+0.07}$ & $17.96_{-0.10}^{+0.11}$ \\
\hline Case 4 & $>100$ & NA & {$[<4.7]$} & $\times$ & $\times$ & 4.1 & NA & $(>5.0),[<5.1]$ & $0.13_{-0.01}^{+0.14}$ & $18.58_{-0.16}^{+0.03}$ \\
\hline Case 5 & $>100$ & NA & {$[<4.7]$} & $\times$ & $\times$ & 3.9 & NA & $(>5.0),[<5.1]$ & $0.02_{-0.02}^{+0.06}$ & $18.59_{-0.06}^{+0.02}$ \\
\hline
\end{tabular}

NotE. - Cases 1-5 are for gas in pure CIE, pure photoionization, photoionization plus collisional ionization, non-equilibrium isobaric cooling, and non-equilibrium isochoric cooling states, respectively. $\delta \chi^{2}$ is the difference of the best-fit $\chi^{2}$ of a joint analysis from that of fitting lines individually. The "NA" symbol indicates that the parameter is not applicable to the model, and the " $\times$ " symbol indicates that the model can be ruled out by the data. Limits within parentheses "()" are obtained in joint analysis, and limits within brackets "[]" are derived in fitting individual lines. Errors are reported at $1 \sigma$ uncertainty. See text for details.

results of simulations and calculations, the metallicity is a known parameter and the ionization corrections can easily be calculated. Once the physical parameters are constrained from spectral fitting, we can infer the column density of total hydrogen. For example, the best acceptable photoionization and hybrid ionization scenarios for the red component indicate that the absorber contains a total $N_{\mathrm{H}}=(0.7-1.2) \times$ $10^{18} \mathrm{~cm}^{-2}$ (Table 2). We are applying this joint analysis technique to systematically study intergalactic absorption lines, constrain the physical properties of the absorbers, and estimate the total baryons contained within the absorbers.

In summary, we have presented a physically-based absorption line model for the spectroscopic study of the IGM. In this model, we can examine the photoionization equilibrium, collisional ionization equilibrium, photoionization plus collisional ionization, and post-shock gas undergoing nonequilibrium isobaric and isochoric cooling. For the WHIM, in which $40-50 \%$ of the baryons at the current epoch are believed to reside, non-equilibrium cooling plus photoioniza- tion should be a more proper scenario; however, such calculations are not yet available in the literature. For the purpose of demonstration, we only simulated a 100 -kpc thick (e.g., Penton et al. 2002) intervening cloud for the absorbing gas along the sight line to 1ES 1553+113. Because our model directly uses the results from calculations and simulations, any progress in future in modeling the WHIM and in simulating the 10-100 kpc scales of the circumgalactic medium can easily be adopted.

The authors have benefited from the discussions with Blair Savage, Anand Narayanan, and Daniel Wang. We also thank the referee for helpful comments and questions. This work at the University of Colorado was partly supported by NASA grant NNX08AC14G for data analysis and scientific discoveries related to the Cosmic Origins Spectrograph on the Hubble Space Telescope, and by NNX07AG77G for theoretical work (JMS). YY also appreciates financial support by NASA through ADP grant NNX10AE86G.

\section{REFERENCES}

Aracil, B., et al. 2006, MNRAS, 367, 139

Arnaud, K. A. 1996, Astronomical Data Analysis Software and Systems V, eds. Jacoby G., Barnes J., ASP Conf. Series, 101, 17

Asplund, M, et al. 2009, ARA\&A, 47, 481

Birnboim, Y., \& Dekel, A. 2003, MNRAS, 34, 349

Buote, D. A., et al. 2009, ApJ, 695, 1351

Cen, R., \& Fang, T. 2006, ApJ, 650, 573

Cen, R., \& Ostriker, J. 2006, ApJ, 650, 560

Danforth, C. W., \& Shull, J. M. 2005, ApJ, 624, 555

Danforth, C. W., \& Shull, J. M. 2008, ApJ, 679, 194 (DS08)

Danforth, C. W., Stocke, J. T., \& Shull, J. M 2010a, ApJ, 710, 613

Danforth, C. W., Keeney, B. A., Stocke, J. T., Shull, J. M., \& Yao, Y. 2010b, ApJ, 720, 976

Davé R., et al. 2001, ApJ, 552, 473

Fang, T., et al. 2002, ApJ, 565, 86

Fang, T., Canizares, R. C., \& Yao, Y. 2007, ApJ, 670, 992

Ferland, G. J., et al. 1998, PASP, 110, 761

Gnat, O., \& Sternberg, A. 2007, ApJS, 168, 213

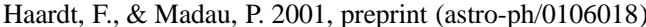

Jenkins, E. B., et al. 2005, ApJ, 623, 767

Kaastra, J. S., et al. 2006, ApJ, 652, 189
Kereš, D., Katz, N., Fardal, M., Davé, R., \& Weinberg, D. H. 2009, MNRAS, 395, 160

Narayanan, A., Savage, B. D., \& Wakker, B. P. 2010, ApJ, 712, 1443

Nicastro, F., et al. 2005, Nature, 433, 495

Oppenheimer, B. D., \& Davé, R. 2008, MNRAS, 387, 577

Penton, S. V., Stocke, J. T., \& Shull, J. M. 2002, ApJ, 565, 720

Penton, S. V., Stocke, J. T., \& Shull, J. M. 2004, ApJS, 152, 29

Pratt, G. W., et al. 2007, A\&A, 461, 71

Rauch, M., et al. 1997, ApJ, 489, 7

Richter, P., Savage, B. D., Tripp, T. M., \& Sembach, K. R, ApJS, 153, 165

Rybicki, G. B., \& Lightman A. P. 1979, Radiative Processes in Astrophysics (New York: Wiley)

Savage, B. D., Lehner, N., Wakker, B. P., Sembach, K. R., \& Tripp, T. M. 2005, ApJ, 626, 776

Shull, J. M. 2009, in Future Directions in Ultraviolet Astronomy. AIPC, 1135,301

Thom, C., \& Chen, H.-W. 2008, ApJ, 683, 22

Tripp, T. M., Sembach, K. R., Bowen, D. V., Savage, B. D., Jenkins, E. B., Lehner, N., \& Richter, P. 2008, ApJS, 177, 39

Yao, Y., \& Wang, Q. D. 2005, ApJ, 624, 751

Yao, Y., et al. 2009, ApJ, 697, 1784

Yao, Y., Wang, Q.-D., Penton, S. V., Tripp, T. M., Shull, J. M., \& Stocke, J. T. 2010, ApJ, 716, 1514 